\title{
The Wardle Instability in Interstellar Shocks: I. Nonlinear Dynamical Evolution
}

\author{
James M. Stone \\ Department of Astronomy, University of Maryland, College Park MD 20742 \\ jstone@astro.umd.edu
}

Received — 


\begin{abstract}
The nonlinear evolution of unstable C-type shocks in weakly ionized plasmas is studied by means of time-dependent magnetohydrodynamical simulations. This study is limited to shocks in magnetically dominated plasmas (in which the Alfvén speed in the neutrals greatly exceeds the sound speed), and microphysical processes such as ionization and recombination are not followed. Both two-dimensional simulations of planar perpendicular and oblique C-type shocks, and fully three-dimensional simulation of a perpendicular shock are presented.

For the cases studied here, the instability results in the formation of dense sheets of gas elongated in the direction of shock propagation, and oriented perpendicular to the magnetic field. The formation of a weak J-type front is associated with the growth of the instability from an equilibrium shock structure. After saturation, the magnetic field structure consists of arches which bow outwards in the direction of shock propagation, and which are anchored by the enhanced ion-neutral drag in the dense sheets. In analogy to the magnetic buoyancy (Parker) instability, saturation occurs when the magnetic tension in the distorted field lines is balanced by drag in the sheets. For the magnetically dominated shocks studied here, the distortions in the magnetic field which produce saturation are very small. Nonetheless, the enhancements of the ion and neutral densities in the sheets is very large, between two and three orders of magnitude compared to the preshock values. At these high densities, recombination processes may be important. The sheets evolve slowly in time, so that shocks propagating in a homogeneous medium may leave behind a network of intersecting filaments and sheets of dense gas elongated in the direction of shock propagation and perpendicular to the mean field. The temperature structure and emission properties of unstable C-type shocks in the nonlinear regime are presented in a companion paper (Neufeld \& Stone 1997).
\end{abstract}

Subject headings: shock waves - instabilities - MHD - methods: numerical - ISM: kinematics and dynamics, magnetic fields 


\section{Introduction}

Shock solutions to the equations of magnetohydrodynamics (MHD) in a weakly ionized plasma have been found to contain a rich variety of structures (Draine \& McKee 1993). Currently, such solutions are classified into three types (Draine 1980; Chernoff 1987). 'J-type' shocks contain one or more discontinuous jumps in the fluid properties, such as density, temperature, or velocity. 'C-type' shock solutions consist of a continuous transition in all fluid variables mediated by ion-neutral drag. ' $\mathrm{C}^{*}$-type' shocks, a new class described by Roberge \& Draine (1990), also consist of a continuous transition in all variables, however in contrast to C-type shocks the downstream neutral gas velocity is subsonic over a portion of the shock structure. Whether a particular shock is J-type, C-type, or $\mathrm{C}^{*}$-type depends upon the shock speed $v_{s}$, and the properties of the ambient medium into which it propagates. Especially important in this respect is the cooling rate of the gas; shocks in gas in which the cooling rate is high enough so that the cooling length is small compared to the ion-neutral coupling length are likely to be C-type. Weak shocks with $v_{s} \lesssim 20-50 \mathrm{~km} \mathrm{~s}^{-1}$ propagating into dense gas with a low ionization fraction are generally thought to be C-type (McKee, Chernoff \& Hollenbach 1984).

Observationally, C-type shocks are of great interest for a number of reasons. Frictional heating of the neutrals associated with the ion-neutral drag can excite strong infrared emission lines (Draine, Roberge, \& Dalgarno 1983; Neufeld \& Melnick 1987; Kaufman \& Neufeld 1996a), or possibly water maser emission (Kaufman \& Neufeld 1996b). At the same time, strong radiative cooling keeps the gas throughout a C-type shock cool enough to prevent the dissociation of molecules. In fact, rather than destroying molecules, the warm temperatures $(T \lesssim 4,000 \mathrm{~K})$ reached by the gas in C-type shocks may instead drive endothermic reactions, potentially making them sites of rich interstellar chemistry. Theoretical models of steady-state planar C-type shocks provide the best fit to the emission observed towards the Kleinman-Low nebula in Orion (Draine \& Roberge 1982; Chernoff, Hollenbach \& McKee 1982; Smith 1991), hot chemistry in molecular clouds (e.g. Hartquist et al 1990), and have been used to account for molecular interstellar absorption lines observed in the spectra of some bright stars (Draine \& Katz 1986).

Interestingly, however, Wardle (1990, hereafter W90; 1991a; b) has shown that planar C-type shock solutions are unstable to perturbations in the shape of the shock front above a threshold neutral Alfvénic Mach number $A \equiv v_{s} / v_{A, n}$ (where $v_{A, n}$ is the Alfvén speed in the neutrals) of $A \approx 5$. The physical mechanism of the instability bears a close resemblance to the magnetic buoyancy (Parker) instability in the galactic disk, with the ion-neutral drag playing the role of gravity. Small amplitude corrugations introduced into the shape of the magnetic field lines can cause the ions to collect in the 'valleys' of the field under the action of the component of drag parallel to the field. The enhanced ion density, and therefore drag, in the 'valleys' causes the perturbation to grow, leading to instability. Through semi-analytic techniques, Wardle found the maximum growth rate of this instability increases rapidly with the shock strength: for an $A=10$ shock it is of order

a few times $t_{\text {flow }}^{-1}$, where $t_{\text {flow }} \equiv\left(\alpha \rho_{i}\right)^{-1}$ is the flow time of ions through the shock ( $\alpha$ is the ion-neutral coupling constant, and $\rho_{i}$ the preshock mass density of ions). The wavelength of the fastest growing mode is of order the shock thickness $L_{s} \equiv v_{A, n} t_{\text {flow }}$. For parameters typical of dense molecular gas, Wardle found $t_{\text {flow }} \approx 10^{3} \mathrm{yrs}$, and $L_{s} \approx 0.01 \mathrm{pc}$. Although Wardle's calculations were unable to predict the structures produced in the nonlinear, saturated stage of the instability, it is reasonable to expect large spatial variations in the ion-neutral drag, and therefore the heating rate of the gas, in comparison to steady-state 
planar solutions. Such variations may have important effects on the emission properties and molecular chemistry in the gas. Without detailed dynamical calculations of the properties of unstable shocks in the nonlinear regime, combined with calculations of the resulting emission properties, the utility of steady-state solutions for planar, high velocity $(A \gtrsim 5)$ C-type shocks in modeling the emission and molecular absorption line observations in dense interstellar gas is uncertain.

The first calculations of the nonlinear stage of the Wardle instability have been presented by Tóth (1994, hereafter T94; 1995). T94 focused on a discussion of suitable numerical algorithms for following the time-dependent growth of the instability into the nonlinear regime. Simulations of three C-type shocks using different parameters confirmed the linear growth rates predicted by Wardle, and showed evidence of large amplitude compression of the ion and neutral densities into structure elongated with the flow. Tóth (1995) used numerical simulations to measure growth rates of the Wardle instability in regions of parameter space not amenable to the techniques used by W90, e.g. unstable wavevectors out of the plane defined by $\mathbf{B}$ and the shock normal. Compression of the flow into dense structures, which at times detach from the shock front, was again noted.

Recently, the ZEUS code (Stone \& Norman 1992a; 1992b) has been extended to allow the study of the dynamics of partially ionized plasmas. This new version of ZEUS, hereafter referred to as ZEUS-2F, implements a numerical algorithm which solves the full set of conservation laws for the coupled ion and neutral fluids, including time-implicit differencing of the collisional drag terms. Although MacLow et al (1995) have described a method for incorporating the effects of ambipolar diffusion into the ZEUS code, their extension is applicable only in the strong coupling limit, and assuming ionization equilibrium holds throughout (so that the ion density simply scales as a power of the neutral density). While these assumptions are useful in a wide variety of circumstances, they preclude the study of the Wardle instability in C-type shocks, which motivates the extensions to the ZEUS code described here. (MacLow \& Smith 1997 have independently developed similar extensions to the ZEUS code to study C-type shocks.)

In this paper, ZEUS-2F is used to study the nonlinear evolution in two-dimensions of unstable C-type shocks which propagate in a direction which is either perpendicular or oblique to the ambient magnetic field direction. Moreover, for the first time, a fully three-dimensional simulation of an unstable C-type shock is presented. In each case the evolution is followed far into the nonlinear regime, allowing the saturation mechanism to be identified. In all cases studied, very large density enhancements (by factors of several hundred) in the postshock gas are produced in the nonlinear regime, with corresponding per-particle heating rate enhancements of a factor of a few. In a companion paper (Neufeld \& Stone 1997, hereafter Paper II), the effect of these variations on the temperature distribution and line emission from unstable C-type shocks is examined in detail.

The organization of this paper is as follows. In the following section, the numerical algorithms, methods for computing initial C-type shock structure, and parameters used in the simulations are described. In $\S 3$ both two- and three-dimensional simulations of unstable C-type shocks are presented, while conclusions are made in $\S 4$.

\section{Methods}




\subsection{Numerical Algorithms}

In a partially ionized plasma, the ions and neutrals obey separate systems of conservation laws that are coupled only through a frictional drag term. Following Draine (1980), the evolution equations can be written as

$$
\begin{gathered}
\frac{\partial \rho_{i}}{\partial t}+\nabla \cdot \rho_{i} \mathbf{v}_{i}=0 \\
\frac{\partial \rho_{n}}{\partial t}+\nabla \cdot \rho_{n} \mathbf{v}_{n}=0 \\
\rho_{i}\left(\frac{\partial \mathbf{v}_{i}}{\partial t}+\mathbf{v}_{i} \cdot \nabla \mathbf{v}_{i}\right)=-\nabla P_{i}+\frac{1}{4 \pi}(\nabla \times \mathbf{B}) \times \mathbf{B}+\alpha \rho_{i} \rho_{n}\left(\mathbf{v}_{n}-\mathbf{v}_{i}\right), \\
\rho_{n}\left(\frac{\partial \mathbf{v}_{n}}{\partial t}+\mathbf{v}_{n} \cdot \nabla \mathbf{v}_{n}\right)=-\nabla P_{n}-\alpha \rho_{i} \rho_{n}\left(\mathbf{v}_{n}-\mathbf{v}_{i}\right), \\
\frac{\partial \mathbf{B}}{\partial t}=\nabla \times\left(\mathbf{v}_{i} \times \mathbf{B}\right) .
\end{gathered}
$$

Here, each symbol has its usual meaning with the subscript $i(n)$ denoting ion (neutral) variables. The collisional coupling constant $\alpha$ is assumed to be a constant; any dependence of $\alpha$ upon the drift velocity, $\left(v_{n}-v_{i}\right)$ is neglected. The strength of the collisional coupling is conveniently characterized by the characteristic shock length scale, $L_{s} \equiv V_{A, n}^{0} /\left[\alpha \rho_{i}^{0}\right]$, where $V_{A, n}^{0}$ is the Alfvén speed in the preshock neutral fluid and $\rho_{i}^{0}$ is the preshock density of the ionized fluid. A flow timescale may also be defined, $t_{\text {flow }} \equiv L_{s} / V_{s}=1 /\left[\alpha \rho_{i}^{0} A\right]$, where $A$ is the Alfvén Mach number for the neutral fluid. Note that $\alpha$ appears only as the product $\alpha \rho_{i}$ in [3] and [4].

There are a number of assumptions which have been made in writing equations [1]-[5]. Firstly, ionization and recombination processes are neglected, so that there are no source or sink terms in equations [1]-[2]. This assumption will be valid in the linear regime provided the growth time of the instability is short compared to ionization and recombination times. However, in regions of very high density formed in the nonlinear stage of the instability, this assumption may break down. Including a realistic treatment of ionization and recombination is difficult due to uncertainties in the dominant microphysical processes themselves, and because dynamical simulations at a realistic value of the ion fraction in dense gas (i.e. $\rho_{i} / \rho_{n} \lesssim 10^{-7}$ ) are very difficult. The potential effects of recombination on the saturation of the instability are discussed more fully in $\S 3$. A second assumption is the use of an isothermal equation of state for both the ions and neutrals, so that $P_{i} / \rho_{i}=P_{n} / \rho_{n}=C_{s}^{2}$, where $C_{s}$ is the isothermal sound speed. This avoids requiring a detailed treatment of the complex cooling processes in the molecular gas, however this assumption limits this study to shocks in magnetically dominated gases, i.e. $V_{A, n} \gg C_{s}$ in the preshock gas (in fact, this condition holds in most regions of the ISM), so that gradients of the thermal pressure are negligible compared to drag and Lorentz forces. In Paper II, calculations of the microphysical molecular cooling processes are performed to compute the equilibrium temperature of gas in the nonlinear stage of the models presented here: these calculations show that even in the nonlinear regime thermal pressure gradients are negligible. Studies of 
the dynamics of shocks in weakly magnetized gases, where $V_{A, n} \leq C_{s}$, will require a proper treatment of the thermodynamics of the gas.

Numerical methods are required to study the nonlinear dynamics of unstable C-type shocks: how can such methods be constructed? Ignoring for the moment the collisional drag term in equations (3) and (4), it is clear the neutral evolution equations (2) and (4) are merely the standard equations of hydrodynamics, while the ion evolution equations (1), (3), and (5) are the equations of ideal MHD. Thus, the hydrodynamical algorithms described in Stone \& Norman (1992a) can be used to evolve the neutrals, while the MHD algorithms described in Stone \& Norman (1992b) can be used to evolve the ions, with in both cases the drag term operator split from the solution of the rest of the equations. Because the ion-neutral drag terms potentially are very stiff, explicit time differencing of these terms are limited by what may be a very restrictive stability criterion. Previous authors have chosen different solutions to this problem: in T94 spatial averaging was used to provide stabilizing diffusion, while MacLow et al (1995) used subcycling on the drag terms at a timestep very much smaller than that dictated by the dynamics. Here, time implicit differencing is used to ensure the stiff drag terms are unconditionally stable. Because the drag term does not involve spatial gradients and is linear in the velocities, implicit difference formula are particularly simple in this case. Let $\mathbf{v}_{i}^{n}$ denote the ion velocity at the current time level $n$, while $\mathbf{v}_{i}^{n+1}$ denote the value at the advanced time level $n+1$ (with $\mathbf{v}_{n}^{n}$ and $\mathbf{v}_{n}^{n+1}$ denoting similar quantities for the neutrals). Then, it is straightforward to show that

$$
\begin{aligned}
& \mathbf{v}_{i}^{n+1}=\frac{\mathbf{v}_{i}^{n}+\alpha \Delta t\left(\rho_{i} \mathbf{v}_{i}^{n}+\rho_{n} \mathbf{v}_{n}^{n}\right)}{1+\alpha \triangle t\left(\rho_{i}+\rho_{n}\right)} \\
& \mathbf{v}_{n}^{n+1}=\frac{\mathbf{v}_{n}^{n}+\alpha \triangle t\left(\rho_{i} \mathbf{v}_{i}^{n}+\rho_{n} \mathbf{v}_{n}^{n}\right)}{1+\alpha \triangle t\left(\rho_{i}+\rho_{n}\right)}
\end{aligned}
$$

where $\triangle t$ is the timestep. Note that due to operator splitting, the ion and neutral densities are both held fixed during the updates [6]-[7]. In the limit of a very large timestep, [6] and [7] converge to the correct equilibrium solution: the final velocities become the density weighted averages of the initial values. Implicit techniques have also been adopted by Tóth (1995).

Numerical algorithms for the solution of equations (1) - (5) including the implicit differencing of the drag terms equations $(6)-(7)$ have been implemented in the new code ZEUS-2F. This code has been subjected to a wide battery of tests. By setting $\alpha=0$, equations (1) - (5) become decoupled so that the hydrodynamic test suite of Stone \& Norman (1992a) can be applied to the conservation laws for the neutrals, while the MHD test suite of Stone \& Norman (1992b) and Stone et al (1992) can be applied to the conservation laws for the ions. With $\alpha \neq 0$, the code has been tested as part of this work by checking that it holds and converges to a steady one-dimensional solution for a C-shock (see $\S 2.3$ ), and that it reproduces the linear growth rates for the instability computed by W90 (see §3.1). As part of a separate investigation of the local dynamics of unstable partially ionized accretion disks, the code also has been shown to reproduce the analytic growth rates of the Balbus-Hawley instability in a partially ionized disk (Hawley \& Stone 1997).

\subsection{Initial Equilibrium Shock Structure}


Simulations of the evolution of unstable C-shocks must begin from an initial equilibrium shock structure. These are calculated directly from equations (1)-(5) assuming a steady-state one-dimensional flow. Let the superscript 0 denote the upstream (preshock) value of a variable. Then, in steady-state, the structure of a C-shock as a function of position $x$ is given by solving

$$
\begin{gathered}
\frac{\partial v_{i x}}{\partial x}=\frac{-\alpha \rho_{n} v_{i x}\left(v_{i x}-v_{n x}\right)}{\left(v_{i x}^{2}-B_{y}^{2} / \rho_{i}-C_{s}^{2}\right)}, \\
\frac{\partial v_{i y}}{\partial x}=\frac{-\alpha \rho_{n} v_{i x}\left(v_{i y}-v_{n y}\right)-\frac{B_{x} B_{y}}{\rho_{i}} \frac{\partial v_{i x}}{\partial x}}{v_{i x}^{2}-B_{x}^{2} / \rho_{i}} \\
\frac{\partial v_{n x}}{\partial x}=\frac{\alpha \rho_{i} v_{n x}\left(v_{i x}-v_{n x}\right)}{\left(v_{i x}^{2}-C_{s}^{2}\right)} \\
\frac{\partial v_{n y}}{\partial x}=\frac{\alpha \rho_{i}\left(v_{i y}-v_{n y}\right)}{v_{n x}},
\end{gathered}
$$

where it can be shown that

$$
\begin{gathered}
\rho_{i}=\rho_{i}^{0} v_{i x}^{0} / v_{i x}, \\
\rho_{n}=\rho_{n}^{0} v_{n x}^{0} / v_{n x}, \\
B_{y}= \begin{cases}B_{y}^{0} \rho_{i} / \rho_{i}^{0} & \text { if } B_{x}=0 \\
B_{x}^{0} v_{i y} / v_{i x} & \text { if } B_{x} \neq 0 .\end{cases}
\end{gathered}
$$

Equations (8) - (14) represent a coupled system of ordinary differential equations that can be integrated using standard techniques. A fourth-order Runge-Kutta algorithm is used in this work (see also T94). For a shock propagating in the $+x$ direction, integration is begun at large $x$ (a few times $L_{s}$ ) with all variables set to their preshock values, and the system (8)-(14) is then integrated backwards through the shock until all slopes are zero. The numerical integration must be started by adding a small value $\delta \ll 1$ to the preshock neutral velocity $v_{n x}^{0}$; here $\delta=10^{-6}$ is used. Solutions to the equilibrium shock structure are specified by choosing values for the four dimensionless parameters: (1) the ion-neutral mass fraction $f=\rho_{i} / \rho_{n}$, (2) the sonic Mach number $M=v_{n x}^{0} / C_{s}$, (3) the Alfvén Mach number $A=v_{n x}^{0} / V_{A, n}^{0}$, and (4) the angle between the shock normal and the magnetic field in the upstream gas $\theta=\arctan \left(B_{y}^{0} / B_{x}^{0}\right)$. Values for these parameters used in this study are discussed in $\S 2.3$.

Once the structure of a steady C-shock is computed using the above techniques, it is used as the initial conditions for a time-dependent evolution computed with the ZEUS-2F code. Typically, a numerical grid of size $-3 L_{s} \leq x \leq 3 L_{s}$ and $0 \leq y \leq 3 L_{s}$ is used, with the shock initially located at $x=0$. The standard numerical resolution is 128 by 64 grid points in each dimension. The three-dimensional simulations use the same $x$ and $y$ domain, with $0 \leq z \leq 3 L_{s}$ in the third dimension. Periodic boundary conditions are applied in the $y$ (and $z$ ) directions, with an inflow boundary (all variables held fixed at the upstream values) at large $x$ and an outflow boundary (zero slope) at small $x$. The preshock medium is given velocity $-v_{s}$, so that the shock structure remains stationary with respect to the grid, i.e. the simulations are carried out in the shock frame. Since C-type shocks are sub-Alfvénic 
in the ions, it is important to ensure the $x$ domain is large enough so that Alfvén waves in the ions reflected off the upstream boundary are damped before they can affect the shock structure. As a test, simulations using a domain of extent $-10 L_{s} \leq x \leq 10 L_{s}$ have been performed; no discernible differences between the structures which arise on this larger domain with those presented here are found.

If the initial shock structure is not perturbed, the ZEUS-2F code will hold the solution arbitrarily long with a maximum relative error in any variable less than $4 \%$ using 32 grid points per shock scale length $L_{s}$. (This error is dominated by a small phase error which causes the shock to move a fraction of a zone with respect to its initial position.) Moreover, the errors converge quadratically with increasing grid resolution as expected for the spatially second-order algorithms used in the ZEUS-2F code. The ability of the code to hold a steady solution, and the convergence of the errors with resolution serve as both a test of ZEUS-2F, and as a check on the numerical integration of (8) - (14).

To seed the Wardle instability, linear amplitude perturbations must be added to the upstream gas. Both random zone-to-zone perturbations of the neutral density with maximum amplitude $\delta \rho_{n}^{0} / \rho_{n}^{0}=10^{-3}$, and a cosine perturbation with the same amplitude and a wavelength equal to the transverse size of the grid are used in the simulations described here. There is no discernible difference in the nonlinear evolution of unstable shocks seeded with random versus cosine perturbations. Because of the isothermal equation of state adopted here, these density perturbations will introduce velocity perturbations of similar amplitude, ensuring all MHD wave modes are seeded initially.

\subsection{Parameters}

Time-dependent solutions to equations (1)-(5) are specified by the three dimensionless quantities: $A, M$, and $\theta$. Conditions in interstellar clouds considerably restrict the allowable range of $A$ and $M$. Given typical neutral Alfvén speeds $\sim 2 \mathrm{~km} \mathrm{~s}^{-1}$ in the dense ISM (Heiles et al. 1993), detailed studies of steady-state C-type shocks (Draine, Roberge \& Dalgarno 1983; Kaufman \& Neufeld 1996a) have shown that the maximum shock velocity for a C-type shock is $\sim 40 \mathrm{~km} \mathrm{~s}^{-1}$, corresponding to a maximum Alfvén Mach number in the neutral fluid $A \sim 20$ ). Faster shocks are of J-type, due to the effects molecular dissociation or self-ionization. Moreover, the linear analysis presented by W90 shows that only shocks with $A>5$ are unstable, so the range of Alfvén Mach number of present interest is $5 \leq A \leq 20$. Typical gas temperatures in the dense ISM are at least $10 \mathrm{~K}$, corresponding to a sound speed $\sim 0.2 \mathrm{~km} \mathrm{~s}^{-1}$, so the relevant range of $M$ is $M \leq 200$. Accordingly, C-type shocks described by the parameter set $A=10$ or $20, M=40,100$, or 200 ; and $\theta=\pi / 2, \pi / 4$, or $\pi / 6$, have been studied.

Our fiducial model with $M=100$ and $A=10$ applies to the case of a $\sim 20 \mathrm{~km} \mathrm{~s}^{-1}$ shock propagating in gas of sound speed $\sim 0.2 \mathrm{~km} \mathrm{~s}^{-1}$ and Alfvén speed $\sim 2 \mathrm{~km} \mathrm{~s}^{-1}$. Given a preshock density of $10^{5}$ particles per $\mathrm{cm}^{-3}$, the characteristic length scale $L_{s} \equiv V_{A, n}^{0} / \alpha \rho_{i}^{0}=2.68 \times 10^{15} \mathrm{~cm}$ (Kaufman \& Neufeld 1996a; Paper II), and the flow time is $t_{\text {flow }} \equiv L_{s} / V_{s}=430$ yr. Note that fixing the shock length scale effectively fixes the product $\alpha \rho_{i}^{0}$. At these densities, the ion fraction $f \sim 10^{-7}$, however we find once $f<10^{-3}$, so that the ion inertia is unimportant, the dynamical evolution is similar. 
Any set of physical parameters which are described by the same dimensionless parameters values will have the same evolution. Thus, the results presented here can be trivially scaled to, for example, a $40 \mathrm{~km} \mathrm{~s}^{-1}$ shock provided the Alfvén and sound speeds in the ambient gas are $V_{A, n}=4 \mathrm{~km} \mathrm{~s}^{-1}$ and $C_{s}=0.4 \mathrm{~km} \mathrm{~s}^{-1}$ respectively. Moreover, the solutions computed here are can also be scaled to the conditions representative of diffuse HI gas. For example, HI gas with $T=20 \mathrm{~K}, n_{H}=100 \mathrm{~cm}^{-3}, B=7.5 \mu \mathrm{G}$, and $V_{s}=20 \mathrm{~km} \mathrm{~s}^{-1}$ result in $M=100$ and $A=10$, i.e. the fiducial model.

\section{Results}

\subsection{Two-Dimensional Perpendicular Shocks}

As a fiducial model of an unstable perpendicular C-type shock, a simulation with parameter values $M=100, A=10$, and $\theta=\pi / 2$ will be discussed. The simulation is evolved to a time of $9 t_{\text {flow }}$ using the standard resolution of $128 \times 64$ zones. Figure 1 plots grayscale images of the ion and neutral densities at five times during the transition to the nonlinear regime. At $t=4 t_{\text {flow }}$ dense clumps can be seen to develop in the ions, while the neutrals show large amplitude perturbations in the shock front. These clumps quickly develop into very thin sheets elongated in the direction of the flow. Dense sheets develop in the neutrals at the same location. By $t=7 t_{\text {flow }}$, the maximum density in these sheets is a factor of 280 times larger than the initial postshock density in the ions, and a factor of 120 times larger in the neutrals. These values are substantially higher than the compression in the initial steady-state shock, which is limited to $\sqrt{2} A$.

Figure 2 plots the time development of the energy associated with the longitudinal component of the magnetic field, normalized by the initial field energy. The plot shows the instability follows a remarkably exponential growth phase from $t=1 t_{\text {flow }}$ to $t=4 t_{\text {flow }}$. The growth rate of the instability, measured from this graph, is $2.4 t_{\text {flow }}^{-1}$. Interpolation of the growth rates in Table 1 of W90 to the parameter values of the fiducial model gives a rate of about $4.6 t_{\text {flow }}^{-1}$. The roughly factor of two discrepancy between these rates is probably due to the use of periodic boundary conditions in the transverse direction, which restricts the allowed wavelengths. At later times, the instability saturates, with no further field amplification occurring. The saturation amplitude is small, only $0.1 \%$ of the initial field energy. This reflects the fact the upstream gas is magnetically dominated (with $V_{A, n}>C_{s}$ ), so that only a small distortion of the field produces large Lorentz forces and leads to saturation. It is possible that in weakly magnetized gases, thermal pressure gradients will play a larger role in determining the structure in the saturated state, so that the sheets may be substantially thicker (however, as discussed in $\S 2$, in this case a detailed treatment of the cooling processes in the gas is required to treat the thermodynamics accurately).

An interesting structure evident at late times $\left(t>5 t_{\text {flow }}\right)$ in Figure 1 is a narrow shell of dense gas parallel to the shock front at the ends of the sheets. The shell appears only in the neutrals. The sharp jump in neutral (but not ion) density across the shell is 
characteristic of a "J-type" front (Draine \& McKee 1993). Indeed, detailed examination of the profiles of the neutral density and velocity indicate the former increases by a factor of roughly sixteen in about 5 zones, while the latter decreases by about four. For an isothermal equation of state in the neutrals, these transitions are consistent with a J-type front with a sonic Mach number of about four. How does the growth of the Wardle instability in a C-type shock lead to the formation of a J-type front? As ions pool in the valleys of the perturbed field lines, the ion-neutral drag is substantially reduced in the peaks. Neutrals entering the shock near the peaks no longer is decelerated completely by drag before encountering the slow-moving, postshock neutrals that are present in the initial equilibrium state. The interaction of the partially decelerated neutrals with the postshock gas leads to the formation of two J-type fronts, one which propagates upstream and provides the final deceleration of the incoming neutrals, and one which propagates downstream and sweeps up postshock gas. Between these two fronts lies the dense shell evident in Figure 1.

The fact that the evolution of unstable C-type shocks can lead to the formation of J-type fronts is interesting in and of itself. In fact, the formation mechanism outlined above seems generic so that it is likely J-type fronts will be associated with the evolution of all unstable C-type shocks, at least in magnetically dominated gases. However, give the small Mach number of the J-type transitions which result (only 0.04 of the sonic Mach number of the original C-type shock in the case of the fiducial model), it is unlikely these fronts will contribute significantly to the total radiative emission from unstable shocks. For example, in the fiducial model, a Mach 4 J-type front will correspond to a propagation speed of only $0.8 \mathrm{~km} \mathrm{~s}^{-1}$. Emission models from the C-type shocks modeled here are described in Paper II.

By $t=7 t_{\text {flow }}$, the transient J-type fronts and associated dense shell are advected off the grid, and a quasi-steady state structure is established. Figure 3 plots the structure of shock at $t=7 t_{\text {flow }}$, deep in the nonlinear regime, in more detail. Figures $3 \mathrm{a}$ and $3 \mathrm{~b}$ are contour plots of the ion and neutral density. Figures $3 \mathrm{c}$ and $3 \mathrm{~d}$ show the instantaneous ion and neutral streamlines. Figure 3e plots representative magnetic field lines, and Figure 3f plots the per-particle heating rate in the neutrals, $H \equiv \rho_{i} / \mu\left(v_{i}-v_{n}\right)^{2}$, where $\mu$ is the mean mass per particle. These plots reveal a variety of interesting structures. The contour plots of the ion and neutral densities reproduce the structure shown in Figure 1. The ion and neutral streamlines show, in both cases, the flow is focused towards the dense sheets beyond their tips. The focusing of the ions is stronger than the neutrals, although in both cases the streamlines remain very smooth. Note that instantaneous streamlines will show the correct orientation of the flow, but give no information about speed. Moreover, no meaning should be assigned to the density of streamlines, the plotting package assigns lines automatically. The plot of magnetic field lines shows both the compression of the field by the shock (indicated by an increase in the density of field lines from right to left), and slight perturbations in the field especially at the tips of the sheets. It is surprising, however, how small the distortions of the field lines are, given the magnitude of the effects in the density (we explore the force balance in both the neutrals and ions quantitatively below and in Figure 4). Finally, the per particle heating rate in the neutrals shows the highest heating rate occurs at the tips of the sheet. Moreover, the maximum value of the heating rate is nearly two times larger than the rate in the initial equilibrium state. This is in indication the temperature in the neutrals may be substantially different in the nonlinear regime of an unstable shock in comparison to a planar steady state model (see Paper II).

An important goal of this study is to identify the saturation mechanism of the Wardle instability. Figure 4 plots the terms in the ion momentum equation along the dotted line 
shown in Figure $3 \mathrm{a}$ at a time of $t=7 t_{\text {flow }}$. Note this location is chosen to intersect the sheets at their tips, where from Figure $4 \mathrm{e}$ the distortion of the magnetic field lines is largest. Figure 4a plots the $x$-components of: (1) the ion-neutral drag $\left(\alpha \rho_{i} \rho_{n}\left[v_{i, x}-v_{n, x}\right]\right.$, solid line), (2) the Lorentz force (dotted line), (3) the pressure gradient in the neutrals (dashed line), and (4) the neutral inertia (or equivalently, ram pressure) $\rho_{n} \mathbf{v}_{n} \cdot \nabla \mathbf{v}_{n}$ (long dashed line). Figure $4 \mathrm{~b}$ plots the $y$-components of the same terms. From Figure $4 \mathrm{a}$, in the direction of shock propagation, the drag and Lorentz forces balance in the ions, whereas the drag and inertia terms balance in the neutrals, except at the peaks. Finite grid effects are primarily responsible for the lack of balance at the peaks, especially for the Lorentz force, since to construct the plot simple finite differencing was used rather than the actual force terms computed using the Method of Characteristics algorithm implemented in the ZEUS-2F code. From Figure 4b, in the transverse direction these same terms balance, though not as well (e.g., in the neutrals, the drag and inertia terms are out of balance near the peaks). As discussed below, this lack of balance in the transverse direction leads to a slow, quasi-static evolution of the sheets in the transverse direction. Both ion and neutral pressure gradients are negligible, even in the $y$-direction where the sheets are very narrow. Saturation occurs when magnetic tension forces due to the distortion of field lines balances the enhanced drag in the ion sheets. This result indicates the nonlinear saturation mechanism of the Wardle instability is directly analogous to the saturation mechanism of the Parker instability, with the ion-neutral drag playing the role of gravity. It is important to determine what mechanism determines the thickness of the sheets. From Figure $4 \mathrm{~b}$, the ions are compressed primarily by the pinch associated with the distortion of the field lines. A resolution study of the fiducial model (using 16, 32, and 64 grid points per shock length $L_{s}$ ) shows the sheets are no more than 2-4 zones wide at each resolution, suggesting their transverse structure is not resolved. Physically, pressure gradients can be expected to set a minimum thickness to the sheets provided $C_{s} \gtrsim V_{A, n}$ in the sheets. If the transverse magnetic field is compressed by a factor $C$ in the shock, this requires a density enhancement of $\sim C^{2}(M / A)^{2} \sim 100 C^{2}$ in the fiducial model. If the sheets are to be resolved with $N$ zones in the transverse direction in the final state, $100 C^{2} \times N$ zones in the transverse zones are required initially (assuming the density enhancement is a result of transverse motion). For any reasonable value of $N$, the resulting numerical resolution required in the initial state greatly exceeds practical limits, suggesting that more efficient (e.g. adaptive grid) techniques are required to resolve the internal structure of the sheets. Thus, the maximum density compressions reported for the simulations presented here should be considered as lower bounds. Note that despite the fact that it is unlikely the transverse density structure is resolved in these simulations, quantities such as the per-particle heating rate, and pinched geometry of the field lines are resolved (i.e. they extend over many grid zones), and do not change with resolution. (For example, with a resolution of $64 \times 32$ zones, the structure and evolution of the sheets is similar to the standard resolution, with the maximum heating rate at the tip of the sheets a factor of $20 \%$ smaller.) Finally, the extreme compressions expected in the sheets in magnetically dominated shocks may increase the effects of recombination in the sheets. In fact, the ion density in, and therefore structure of, the sheets at saturation may be determined by microphysical effects, thus it will be important to incorporate them before pursuing higher resolution simulations.

Note from Figure $4 \mathrm{~b}$ that the net forces in the transverse direction do not balance completely, indicating the structure observed in Figure 1 are not a true steady state. Indeed, when the fiducial model is evolved to $t=9 t_{\text {flow }}$, the two sheets migrate towards one another and finally merge. At the same time, a new sheet forms roughly one $L_{s}$ away (roughly the spacing between the sheets observed in figure 1). In the lab frame, the density 
structure left by the propagation of an unstable C-shock will therefore consist of a network of intersecting filaments, rather than purely parallel sheets. It is probable that the periodic boundary conditions adopted in the transverse direction affect the spacing of the sheets, by restricting the range of available wavenumbers. Calculation of the exact pattern of the density structures left behind by a propagating C-type shock will require simulations with much larger transverse dimensions.

To study the effect of varying the sonic Mach number $M$ on the nonlinear stage of the Wardle instability, simulations of perpendicular C-type shocks with values of $M=40$ and 200 have been performed. In all cases, varying $M$ does not change the structures produced in the nonlinear regime qualitatively. Saturation occurs when the ions and neutrals are compressed into dense sheets elongated with the flow, although since the growth rate of the instability increases with $v_{s}$, saturation occurs at earlier times. For runs using the standard resolution, the maximum density in the ions and neutrals is within a factor of two of that observed for the fiducial model. Moreover, in each case the maximum heating rate is increased by similar factors (about two to four) compared to the heating rate in the initial equilibrium shock structure.

To study the effect of varying the Alfvénic Mach number $A$ on the solutions, a simulation with $A=20$ and $M=200$ has been performed. Once again, the same sheet-like structures are formed. The maximum densities in the ions and neutrals are within $50 \%$ of the values reported for the fiducial model, and the maximum heating rate is a factor of 3.5 times higher than in the initial equilibrium state. In summary, our limited parameter survey indicates that the nonlinear saturation mechanism does not depend on the parameters of the shock, and moreover the maximum compression and increase in heating rate are within factors of two over a wide range of variation in $M$ and $A$.

\subsection{Two-Dimensional Oblique Shocks}

Figure 5 plots the structure of an unstable oblique C-type shock far in the nonlinear regime. The parameters of this shock are identical to those of the fiducial model, except $\theta=\pi / 6$ now. The simulation is run using the standard numerical resolution of $128 \times 64$ grid points. Figures $5 \mathrm{a}$ and $5 \mathrm{~b}$ are contour plots of the neutral and ion densities. Figures $5 \mathrm{c}$ and $5 \mathrm{~d}$ show instantaneous streamlines in the ions and neutrals respectively. Figure 5e plots representative magnetic field lines, and Figure $5 \mathrm{f}$ is the per-particle heating rate in the neutrals (compare Figure 5 to Figure 3 ). The plot is shown at $t=3.8 t_{\text {flow }}$. Running the simulations to longer times is prohibitive as the ion density becomes exceedingly small in some regions of the domain, leading to divergence of the ion Alfvén speed. It is clear the simulation presented in Figure 5 is well into the nonlinear regime, and therefore serves as a useful example of the structure of unstable oblique shocks. Nonetheless, it is also clear that implicit, or semi-implicit, time integration techniques will be required to study the long term evolution of oblique shocks (Toth 1995).

Qualitatively, the structures formed by the instability in an oblique shock are similar to those observed in the perpendicular shock, e.g. Figure 1. Both the ions and neutrals are compressed into dense sheets elongated in the direction of the flow. The sheets are thicker in the oblique shock, and are slightly curved. A dense shell is evident at the base 
of the sheets in the neutrals, indicative of the formation of a J-type front associated with the initial growth of the instability. The ion and neutral streamlines show convergence towards the sheets; in fact backflow (i.e. transverse motions opposite to the far downstream transverse flow direction) is evident in some locations. Note the neutral streamlines show a sharp kink at the location of the dense shell at the base of the sheets, consistent with the presence of a J-type front at this location. The magnetic field lines show distortions which clearly indicate the dense sheets in the ions and neutrals are located at 'valleys', with 'peaks' in between. The backflow observed in the ion streamlines occurs where the magnetic field has opposite slope compared to the far downstream orientation. Finally, the per-particle heating rate is strongly peaked at the tips of the sheets, similar to the perpendicular shock case.

In an oblique shock, the compression ratio in the initial, steady-state shock is higher than a perpendicular shock of the same Mach number. In the nonlinear regime, the maximum compression produced by the instability in an oblique shock is comparable, though somewhat less, than that reported in $\S 3.1$ for a perpendicular shock. At $t=3.8 t_{\text {flow }}$, the maximum density in the neutrals is a factor of 126 times higher than the preshock value, and a factor of 106 higher for the ions. This leads to a per-particle heating rate which is a factor of 2.3 higher compared to the highest value in the initial shock. Again, such variations may have important consequences on the temperature structure of an unstable oblique shock (see Paper II). It is possible that if the shock were evolved to longer times, the maximum compressions in the ions and neutrals, and maximum heating rate, may increase over the values reported here. As in the case of a perpendicular shock, repeating the simulation at one half the numerical resolution does not lead to substantive changes in the maximum compression, or maximum heating rate reported above.

The pattern of distortion in the magnetic field lines evident in Figure 5e suggest the saturation mechanism of the instability is the same in oblique shocks as perpendicular shocks, i.e. magnetic tension balanced by enhanced drag in the sheets (which acts as an effective gravity that anchors the field lines). Indeed, plots of each component of the forces acting on the ions and neutrals in a horizontal slice through the sheets similar to Figure 4 confirms this expectation: in the ions the Lorentz force is balanced primarily by drag, while in the neutrals drag is balanced by inertia (or ram pressure). For the isothermal gas studied here, numerical resolution plays a role in determining the final thickness of the sheets. However, the level of agreement between the components is worse in the transverse direction than for perpendicular shocks, indicating the structures shown in Figure 5 are not steady. Indeed, the evolution preceding $t=3.8 t_{\text {flow }}$ shown in Figure 5 shows the sheets are in the process of merging into one larger structure. Unfortunately, the difficulty of following the evolution with time explicit techniques prevents the study of structures which emerge thereafter. However, since a similar merger process is observed in perpendicular shocks on longer timescales, and in this case the merged sheet is qualitatively unchanged, there is no reason to expect the merger of sheets in oblique shocks will result in any new behavior. We also note that in addition to merging, the sheets drift transverse to the shock front at about the Alfvén speed, as expected (Wardle 1991b).

In order to examine the effect of varying the obliquity angle, simulations of unstable shocks with $\theta=\pi / 4$ and $\theta=\pi / 3$ have also been performed. Although the maximum compression in the initial equilibrium state changes with obliquity angle, no substantive change in the maximum compression, maximum increase in heating rate, or qualitative structure of the sheets formed in the nonlinear regime are found for the obliquity angles considered here. 


\subsection{A Three-Dimensional Perpendicular Shock}

To study the fully three-dimensional structure of unstable C-type shocks, the fiducial model for a perpendicular shock (with parameter values $M=100, A=10$, and $\theta=\pi / 2$ ) has been evolved on a grid of $188 \times 64 \times 64$ in the domain $-3 L_{s} \leq x \leq 3 L_{s}, 0 \leq y \leq 3 L_{s}$, and $0 \leq z \leq 3 L_{s}$. Because of the computational expense of three-dimensional simulations, a parameter survey was not performed, nor have any oblique shock models been computed in three-dimensions. Instead, this section will describe the results from this single model.

Figure 6a shows an isosurface of the ion density at $t=9.6 t_{\text {flow }}$, using the level $\rho_{i}=0.02\left(\rho_{i}^{0}=0.001\right.$ in the dimensionless units used here). The view is such that the shock propagates from right (rear) to left (forward), and the magnetic field is oriented horizontally (see arrows). A horizontal slice through the three-dimensional volume corresponds to the two-dimensional simulation described in §3.1. Note that as one might expect, the ions have formed dense sheets perpendicular to the magnetic field direction. In fact, the gross morphology of all the dynamical variables are similar to the $2 \mathrm{D}$ results: the neutral also form vertical sheets at the same locations as those in the ions, and, in addition, there is a dense shell of neutrals located at the base of the sheets associated with J-type fronts. Moreover, the ion and neutral streamlines in the horizontal plane show focusing towards the sheets, and the component of the magnetic field in the horizontal plane are distorted in a similar fashion to that shown in Figure 3e. However, note the sheets are not symmetric in the vertical direction, but the leading edge of the sheet shows large variations in $x$-position. Moreover, the sheets are not perfectly planar, but are slightly folded in the horizontal direction (this is best traced along the rear edges of the sheets), i.e. the $y$-coordinate of the leading edge of the sheet varies with $z$. Both of these patterns are evidence of vertical modes.

Figure $6 \mathrm{~b}$ plots an isosurface of the per-volume heating rate at the same time as the view in Figure $6 \mathrm{a}$, and at the level $H=20$ (the maximum heating rate in the initial state is $H^{0}=5.9$ ). Unlike the two-dimensional case, where the heating rate is strongly localized to the tip of the sheets, in three-dimensions there is significant heating in small regions throughout the volume of the sheets. Significant heating in horizontal strips along the tips of the sheets is evident.

The complex pattern of the heating rate is an indication that motions in the third dimension cause significant variations in the values of flow variables compared to the two-dimensional flow field. Indeed, at $t=9.6 t_{\text {flow }}$, the maximum value of the neutral density is a factor of 339 times higher than the preshock neutral density, which is significantly higher than the maximum value in two-dimensions. The difference in the ion density is even more pronounced: the maximum ion density is 772 times higher than the preshock value, nearly an order of magnitude higher than the result in two-dimensions. These values lead to a dramatic increase in the maximum heating rate, a factor of 30 times higher than the steady-state value. It is likely that the variation in temperature structure in a 3D shock is even more pronounced than in 2D (see Paper II). At the same time, the high compressions in the postshock gas (nearly three orders of magnitude compared to the preshock gas in the case of the ions) indicates the need to incorporate microphysical processes such as recombination to correctly model the saturated structure.

Finally, in Figure 7 the time development of the magnetic energy associated with the components of the field perpendicular to the initial field direction $\left(B_{x}\right.$ and $\left.B_{z}\right)$ is plotted. The growth rates of these components is generally lower than that measured in the $2 \mathrm{D}$ 
simulation (cf. Figure 2). The time evolution of both components are nearly identical, although $B_{x}^{2}$ (the energy density associated with the field component in the direction of shock propagation) is roughly a factor of 10 larger throughout. An exponential growth phase between $t=5 t_{\text {flow }}$ and $8 t_{\text {flow }}$ dominates both components; the measured growth rate during this phase is $1.9 t_{\text {flow }}^{-1}$. This value is somewhat lower than the $2 \mathrm{D}$ result. Saturation again occurs at low amplitude, $0.1 \%$ of the initial magnetic energy.

\section{Conclusions}

Time-dependent MHD simulations have been used to study the nonlinear evolution of the Wardle instability in C-type shocks in both two- and three-dimensions. Shocks which propagate either in a direction perpendicular or oblique to the direction of the magnetic field have been studied. The major conclusions are as follows.

(1) The instability results in the formation of dense sheets of gas elongated in the direction of shock propagation, and oriented perpendicular to the initial direction of the magnetic field. Both the neutral and ion densities are greatly enhanced in these sheets compared to their preshock values, by factors of two to three orders of magnitude in different circumstances. The sheets merge, and new ones are formed, as the shock propagates, leading to the speculation that on large scales, an unstable C-type shock propagating through a homogeneous ambient medium will produce a network of intersecting filaments and sheets of dense gas aligned in the direction of shock propagation and perpendicular to the mean field direction.

(2) Saturation of the instability occurs when magnetic tension in the magnetic field which arches between the sheets is balanced by the enhanced drag that anchors the field in the sheets. In magnetically dominated shocks, the amplitude of the field distortion which produces saturation is very small. For the isothermal equation of state studied here, the final thickness of the sheets is unresolved.

(3) J-type fronts are formed during the initial growth phase of the instability in a planar, steady-state initial shock structure, because of large variations in the ion fraction across the surface of the shock front. It is not clear whether this result will be universally true for the growth of the instability in all initial unstable states. However, because large variations of the ion fraction across the shock seems an inevitable result of the instability, the formation of J-type fronts may be a generic feature of the instability.

(4) The per-particle heating rate of the neutrals is increased by more than a factor of two near the tips of the sheets in two-dimensions, and by a factor of 30 in three-dimensions. Calculations of the temperature and line emission properties of the molecular gas in the

nonlinear stage instability (based on the dynamical models presented here) is examined in Paper II.

It will be important in future work to relax some of the assumptions adopted here. In particular, recombination processes have been neglected, although they may be important in the nonlinear regime due to the extreme compression of the gas into the sheets. Moreover, in order to study shocks in weakly magnetized gas, a realistic treatment of cooling will be 
required rather than the isothermal equation of state used here. Finally, studies of shocks in non-planar geometries (e.g. bow shocks) are important.

I am grateful to David Neufeld and Mark Wardle for stimulating discussions. The computations were performed on the Cray systems at the Pittsburgh Supercomputing Center, and on the CM-5 system at the National Center for Supercomputing Applications. This research was supported by the NSF through grant AST-9528299. 


\section{References}

Chernoff, D.F. 1987. ApJ, 312, 143.

Chernoff, D.F., Hollenbach, D.J., \& McKee, C.F. 1982. ApJ, 259, L97.

Draine, B.T. 1980. ApJ, 241, 1021

Draine, B.T., \& Katz, N. 1986. ApJ, 310, 392.

Draine, B.T., \& McKee, C.F. 1993. ARA\&A, 31, 373.

Draine, B.T., \& Roberge, W.G. 1982. ApJ, 259, L91.

Draine, B.T., \& Roberge, W.G., \& Dalgarno, A. 1983. ApJ, 264, 485.

Hartquist, T.W., Flower, D.R., \& Pineau des Forêts, G. 1990. In Molecular Astrophysics, ed. T.W. Hartquist, Cambridge, CUP, p91.

Hawley, J.F., \& Stone, J.M. 1997. In preparation.

Heiles, C., Goodman, A.A., McKee, C.F., \& Zweibel, E.G. 1993, in Protostars and Planets III, ed. M. Matthews \& E. Levy (Tucson: Univ. of Arizona Press).

Kaufman, M.J., \& Neufeld, D.A. 1996a. ApJ, 456, 611.

Kaufman, M.J., \& Neufeld, D.A. 1996b. ApJ, 456, 250.

MacLow, M.-M., Norman, M.L., Königl, A., \& Wardle, M. 1995. ApJ, 442, 726.

MacLow, M.-M., \& Smith, M. 1997, submitted to ApJ.

McKee, C., Chernoff, D., \& Hollenbach, D. 1984, in Galactic and Extragalactic Infrared Spectroscopy, ed. by M.F. Kessler and J.P. Phillips (Dordrecht: Reidel), 103.

Neufeld, D.A., \& Melnick, G.J. 1987. ApJ, 332, 266.

Neufeld, D.A., \& Stone, J.M. 1997. In preparation (Paper II).

Roberge, W.G., \& Draine, B.T. 1990. ApJ, 350, 700.

Smith, M.D. 1991. MNRAS, 253, 175.

Stone, J.M., \& Norman, M.L. 1992a. ApJS, 80, 753. 
Stone, J.M., \& Norman, M.L. 1992b. ApJS, 80, 791.

Stone, J.M., Hawley, J.F., Evans, C.E., \& Norman, M.L. 1992. ApJ 388, 415.

Tóth, G. 1994. ApJ 425, 171 (T94).

Tóth, G. 1995. MNRAS, 274, 1002.

Wardle, M. 1990. MNRAS, 246, 98 (W90).

Wardle, M. 1991a. MNRAS, 250, 523.

Wardle, M. 1991b. MNRAS, 251, 119. 


\section{Figure Captions}

Figure 1. Time development of the Wardle instability in a $M=100, A=10$ perpendicular C-type shock. The bottom panels are grayscale images of the neutral density at times marked in units of $t_{\text {flow }}=\left(\alpha \rho_{i}\right)^{-1}$. The top panels show the ion density at the same times. The maximum density in the ions (neutrals) is a factor of 280 (120) times larger than the preshock values in the initial, steady-state. The size of the computational domain is $6 L_{s} \times 3 L_{s}$. For a $20 \mathrm{~km} \mathrm{~s}^{-1}$ shock in dense molecular gas, with $L_{s}=2.68 \times 10^{15} \mathrm{~cm}$, $t_{\text {flow }} \approx 430$ yrs.

Figure 2. Time history of the magnetic energy in the longitudinal component (parallel to the shock normal) of the field normalized by the initial field energy for the shock evolution shown in Figure 1. Exponential growth of the instability is evident from $t=1 t_{\text {flow }}$ to $t=4 t_{\text {flow }}$, followed by saturation at a low amplitude.

Figure 3. Structure of a $M=100, A=10$ perpendicular C-type shock in the nonlinear regime, at $t=7 t_{\text {flow }}$. (a) Contours of the ion density between 0.001 and 0.278 . (b) Contours of the neutral density between 1.0 and 119. (c) Instantaneous ion streamlines. (d) Instantaneous neutral streamlines. (e) Representative magnetic field lines. ( $f$ ) Contours of the per-particle heating rate in the neutrals between 0.0 and 55.6. The dashed line in panel (a) is the location of the vertical slice used to construct Figure 4. Note in the dimensionless units quoted here, $\rho_{i}^{0}=0.001, \rho_{n}^{0}=1.0$, and the maximum $H^{0}=22.4$

Figure 4. Plots of various terms in the ion and neutral momentum equations as a function of vertical position $y$ along the slice shown in Figure 3a. This position passes through the regions of maximum heating at the tip of the ion and neutral sheets shown in Figure $3 \mathrm{f}$. Plotted are the ion-neutral drag (solid line), Lorentz force (dotted line), pressure gradient in the neutrals (short dashed line), and neutral inertia term $\rho_{n} \mathbf{v}_{n} \cdot \nabla \mathbf{v}_{n}$ (long dashed line). (a) Terms in the $x$-direction (top panel) (b) Terms in the $y$-direction (bottom panel). Saturation in the ions is a balance between drag and Lorentz forces, while in the neutrals it is a balance of drag and inertia.

Figure 5. Structure of a $M=100, A=10$ oblique C-type shock with $\theta=\pi / 6$ in the nonlinear regime, at $t=3.8 t_{\text {flow }}$. (a) Contours of the ion density between 0.001 and 0.106. (b) Contours of the neutral density between 1.0 and 127. (c) Instantaneous ion streamlines. (d) Instantaneous neutral streamlines. (e) Representative magnetic field lines. 
(f) Contours of the per-particle heating rate in the neutrals between 0.0 and 103 . Note in the dimensionless units quoted here, $\rho_{i}^{0}=0.001, \rho_{n}^{0}=1.0$, and the maximum per-particle heating rate in the initial equilibrium state is 32.5 .

Figure 6. (a) Isosurface $\left(\rho_{i}=0.02\right)$ of the ion density at $t=9.6 t_{\text {flow }}$ in a 3D simulation of a perpendicular C-type shock. The arrows show the directions of the flow velocity and magnetic field. (b) Isosurface $(H=20)$ of the per-volume heating rate. The maximum values of $\rho_{i}$ and $H$ are 339 and 179 respectively.

Figure 7. Time history of the magnetic energy in the components of the field perpendicular to the initial field, normalized by the initial field energy for the 3D shock evolution shown in Figure 6. The solid line plots $B_{x}^{2}$, while the dashed line plots $B_{z}^{2}$. Exponential growth of the instability is evident from $t=5 t_{\text {flow }}$ to $t=8 t_{\text {flow }}$. 


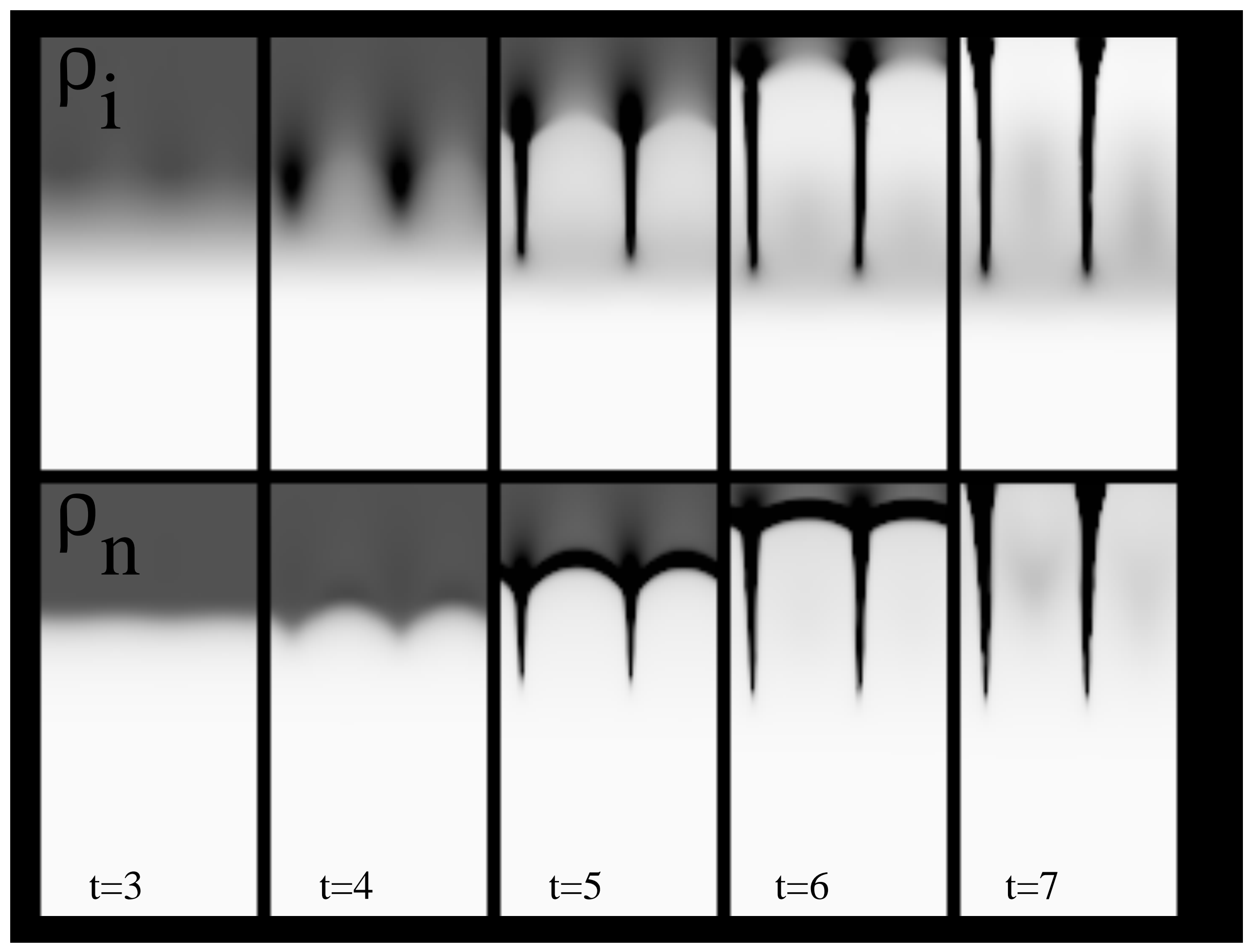




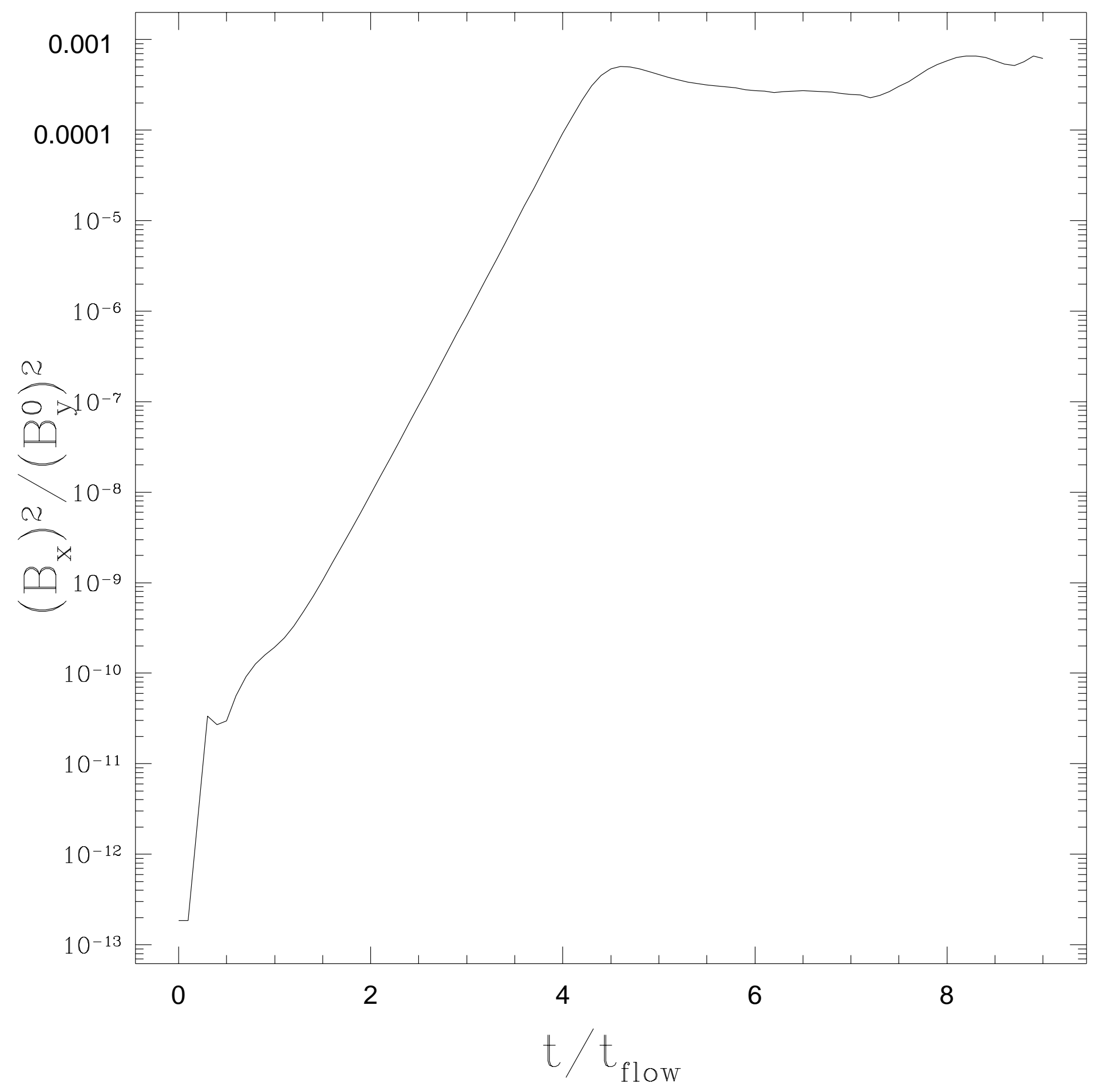



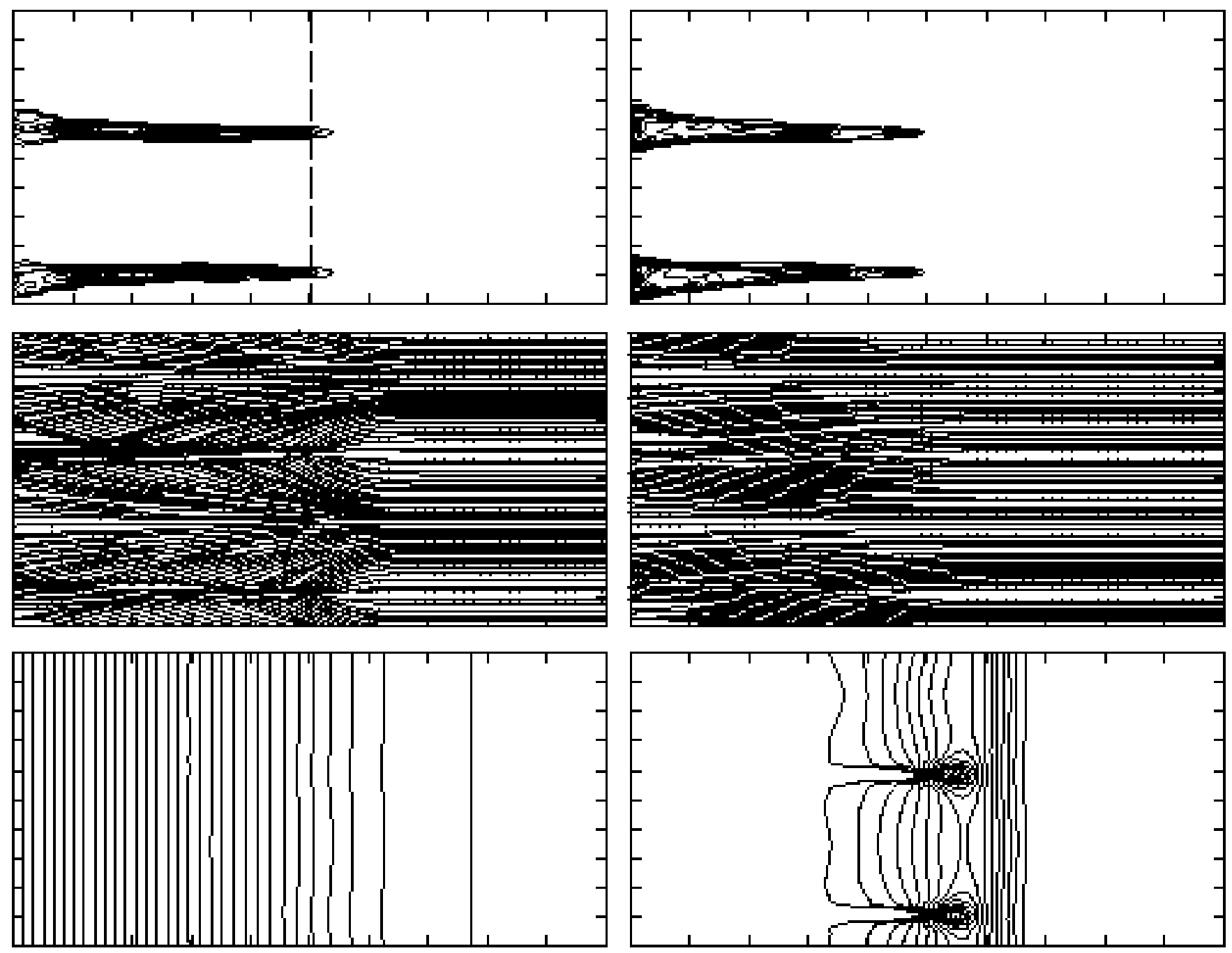

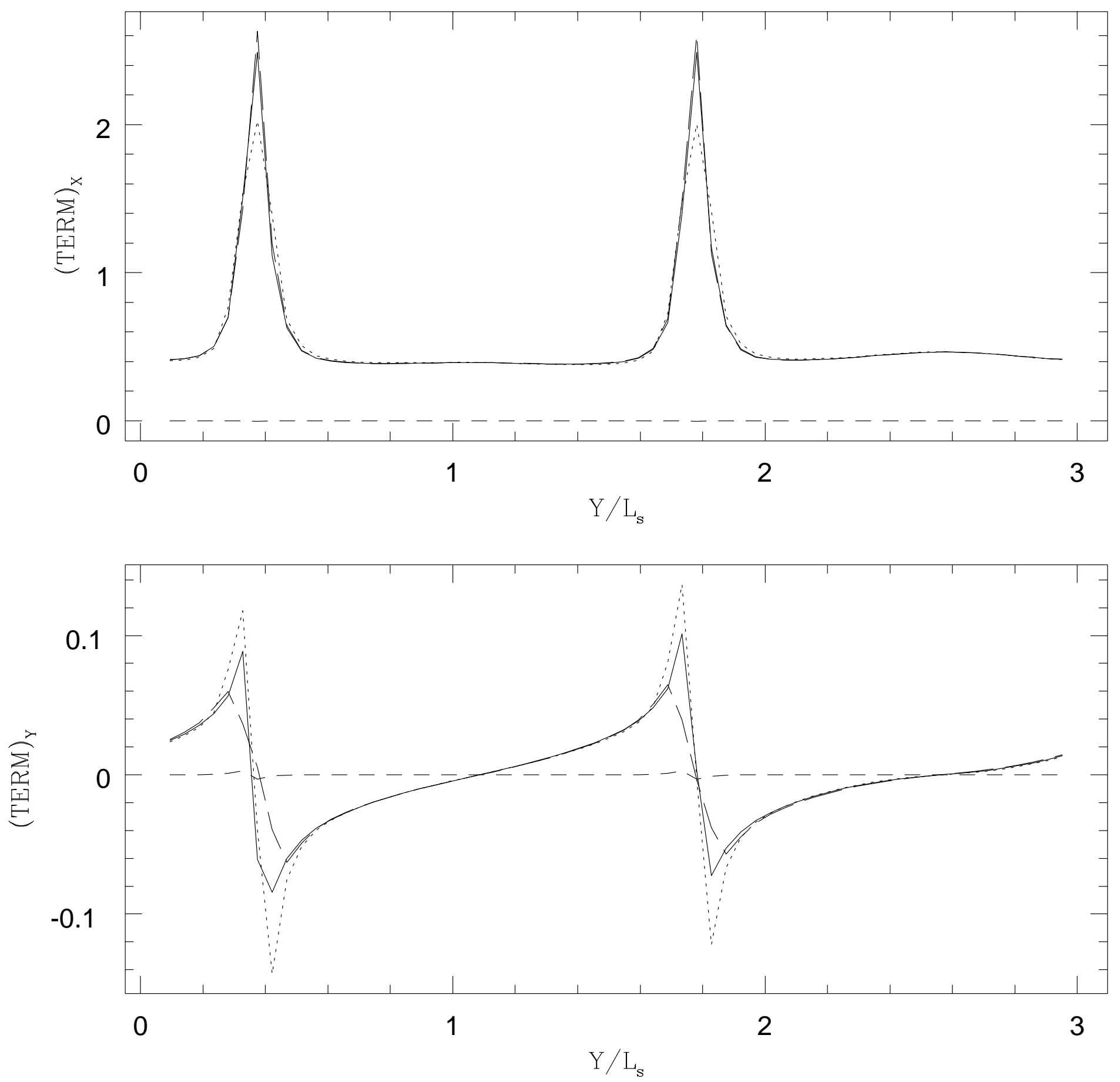

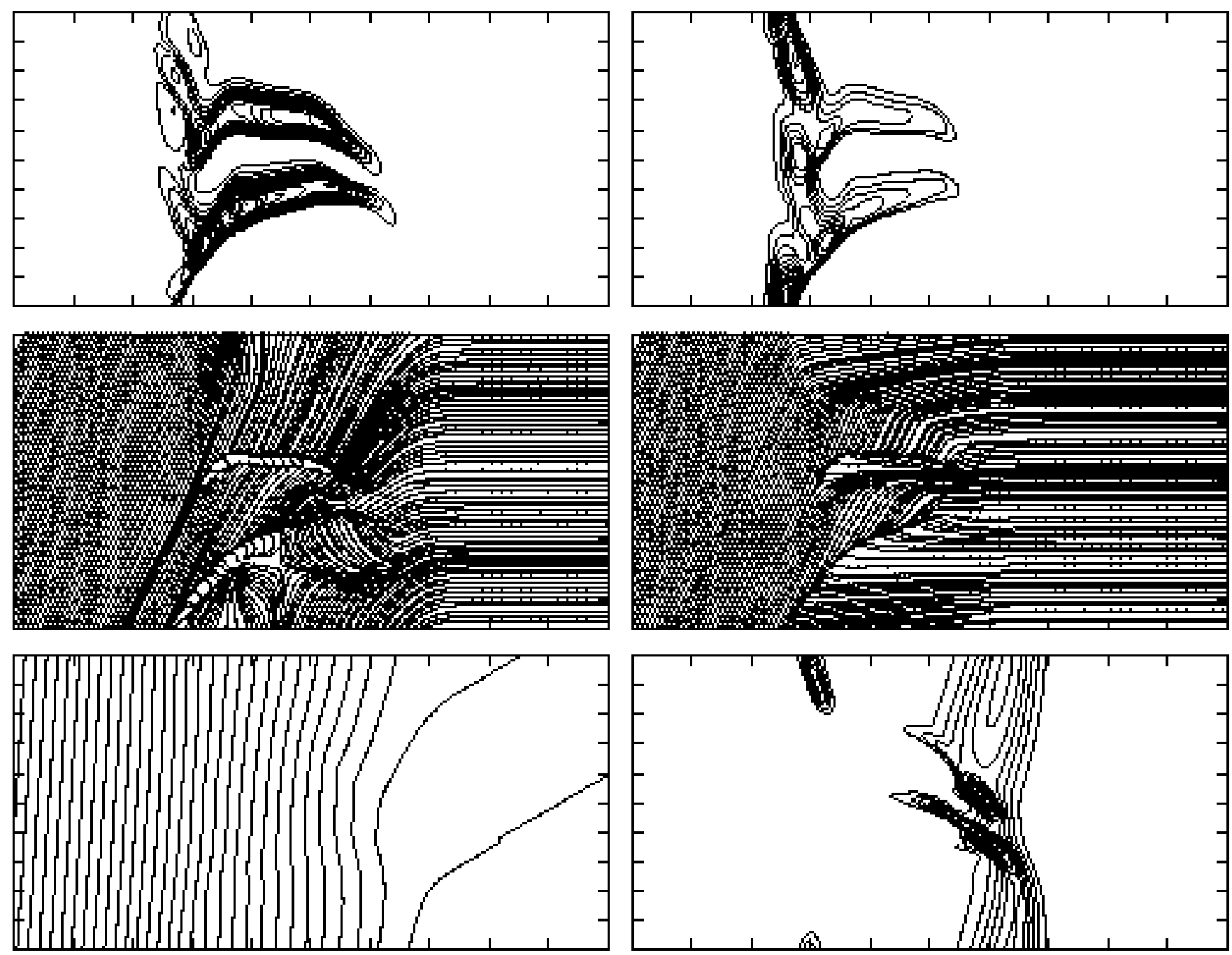


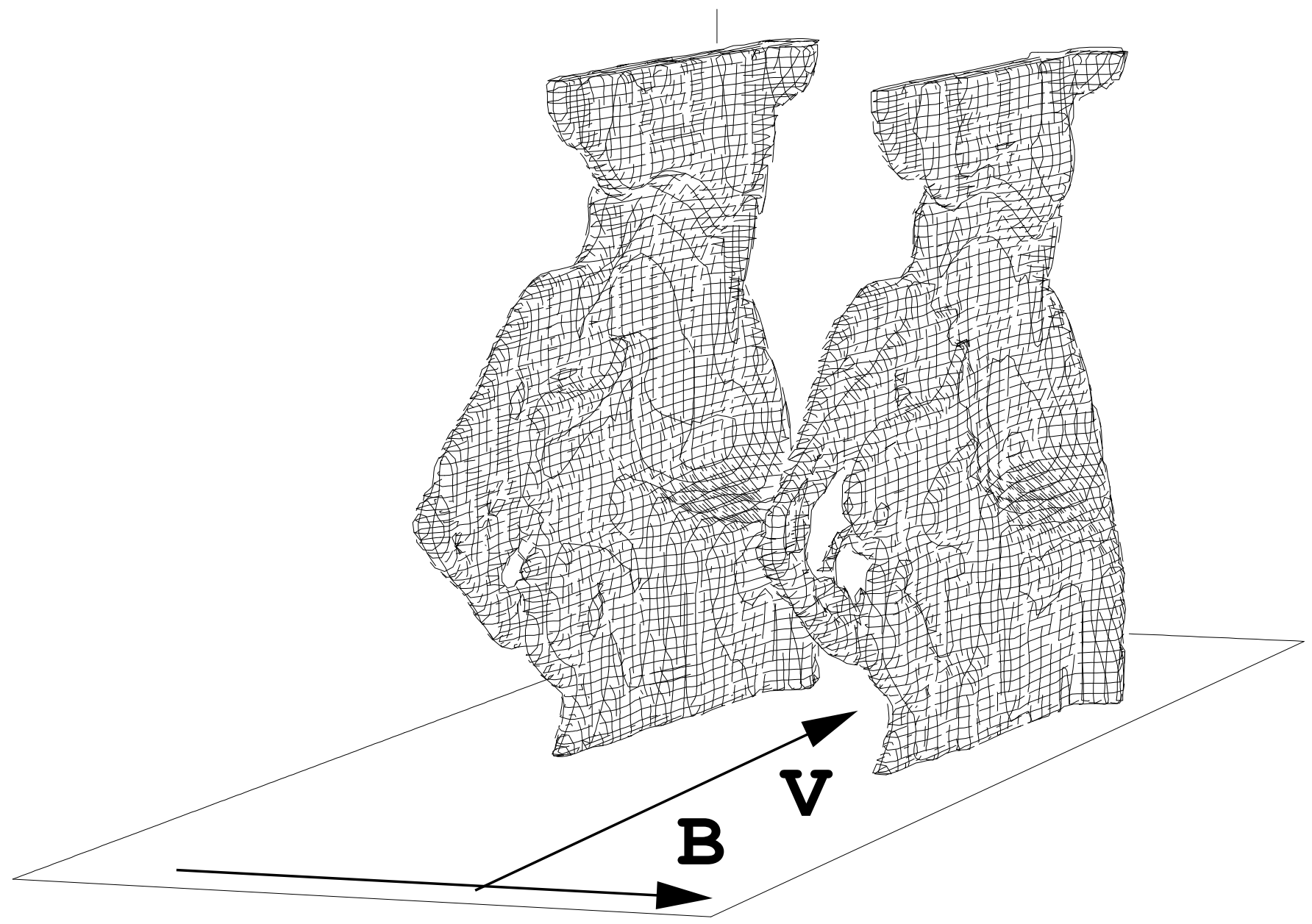




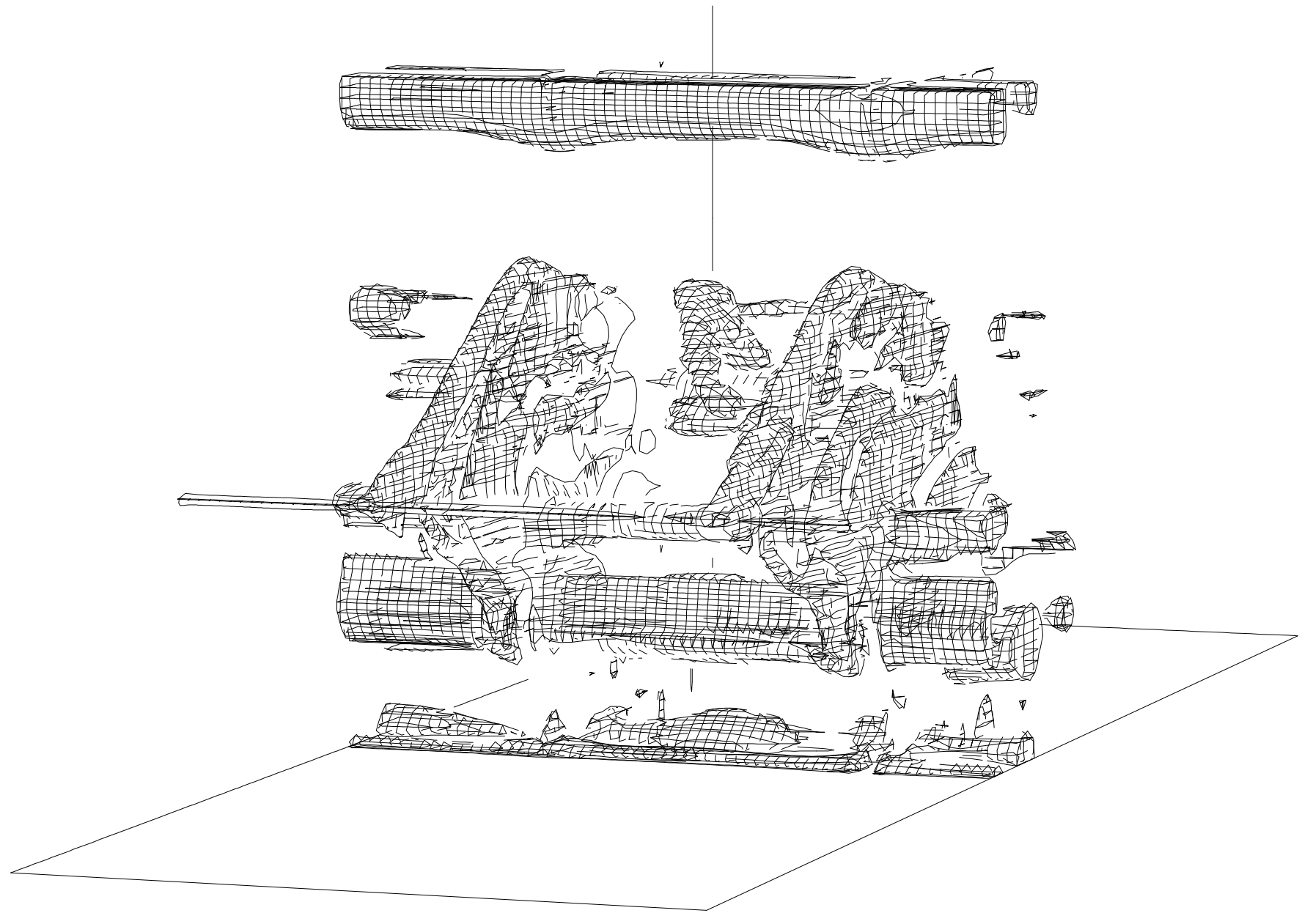




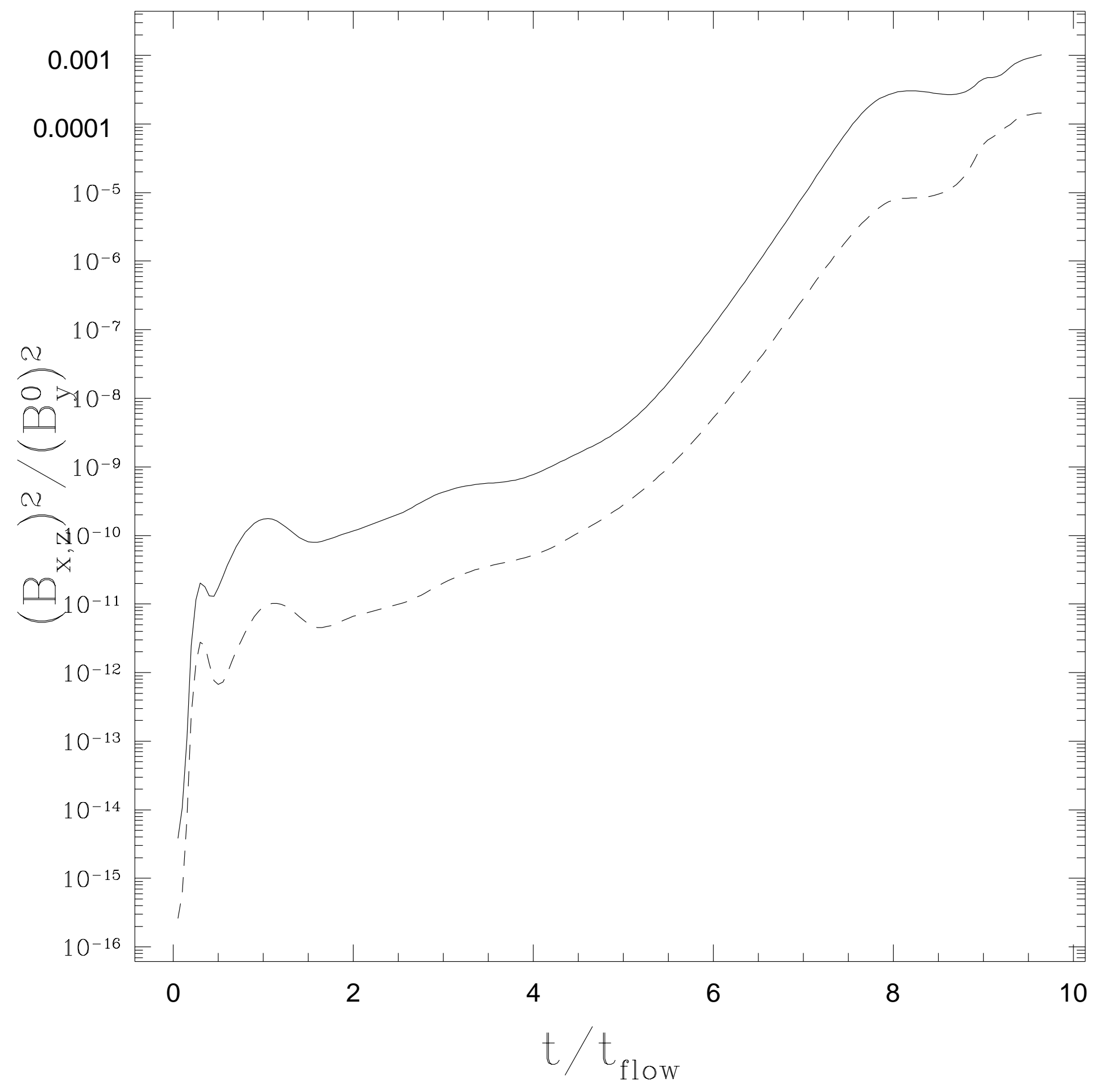

